\title{
Voltage Rollercoaster Filtering of Low Mass Contaminants during Native Protein Analysis
}

\author{
SUPPLEMENTARY MATERIAL
}

\section{RUNNING TITLE: A VOLTAGE ROLLERCOASTER FILTER FOR NATIVE PROTEINS}

John P. McGee ${ }^{\mathrm{a}}$, Rafael D. Melani ${ }^{\mathrm{a}}$, Michael Goodwin ${ }^{\mathrm{b}}$, Graeme McAlister ${ }^{\mathrm{b}}$, Romain Huguet ${ }^{\mathrm{b}}$, Michael W. Senko ${ }^{\text {, }}$ Philip D. Compton ${ }^{\mathrm{a}}$, Neil L. Kelleher ${ }^{*}$

aDepartments of Chemical and Biological Engineering, Chemistry, and Molecular Biosciences, the Chemistry of Life Processes Institute, the Proteomics Center of Excellence at Northwestern University, Evanston, Illinois 60208, United States

${ }^{\mathrm{b}}$ Thermo Fisher Scientific, San Jose, California 95134, United States

Address reprint requests to...

Neil L. Kelleher

2145 Sheridan Rd

Evanston, IL 60208

n-kelleher@northwestern.edu

Tel: $847-467-4362$

Fax: 847-467-3276 


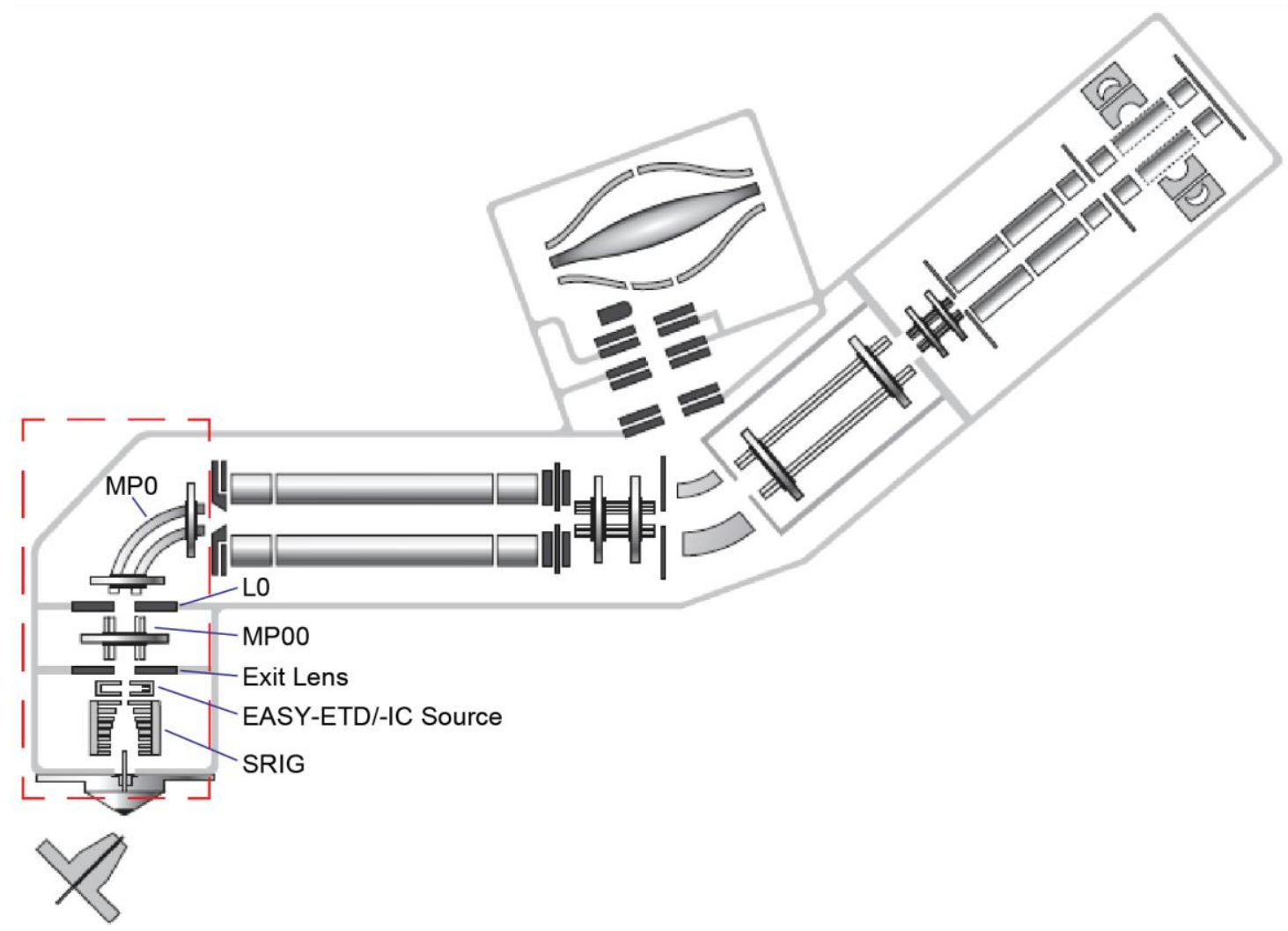

Fig. S1. Hardware schematic of an Orbitrap Tribrid. The region of interest for Voltage Rollercoaster Filtering is boxed in red. The capillary and subsequent pressure transitions allow for entrainment of ions in the inlet gas flow, making the ions' velocities near-uniform and their kinetic energies nonuniform as dictated by their differences in mass. The DC voltages of the optics post-capillary are set in stepped decline, which prevents unintentional ion accumulation. Ions are accelerated from the SRIG to the multipole (MP0), upon which various collisions with background gas reduce the kinetic energies of the ions. The acceleration into the multipole is more drastic given the addition of in-source collisional energy or a higher analyte charge state, and a higher collisional cross-section at the same mass and charge will expose the analyte to more collisions and a larger loss of kinetic energy. The voltage drops across the inter-flatapole lens (L0) guides ions towards the bent flatapole (MP0), and the RF of the flatapole contains the ions as their path turns with the geometry of the flatapole. The exit lens (a vacuum baffle) and EASY-ETD/-IC source are labeled here for clarity but are not manipulated in Voltage Rollercoaster Filtering. 


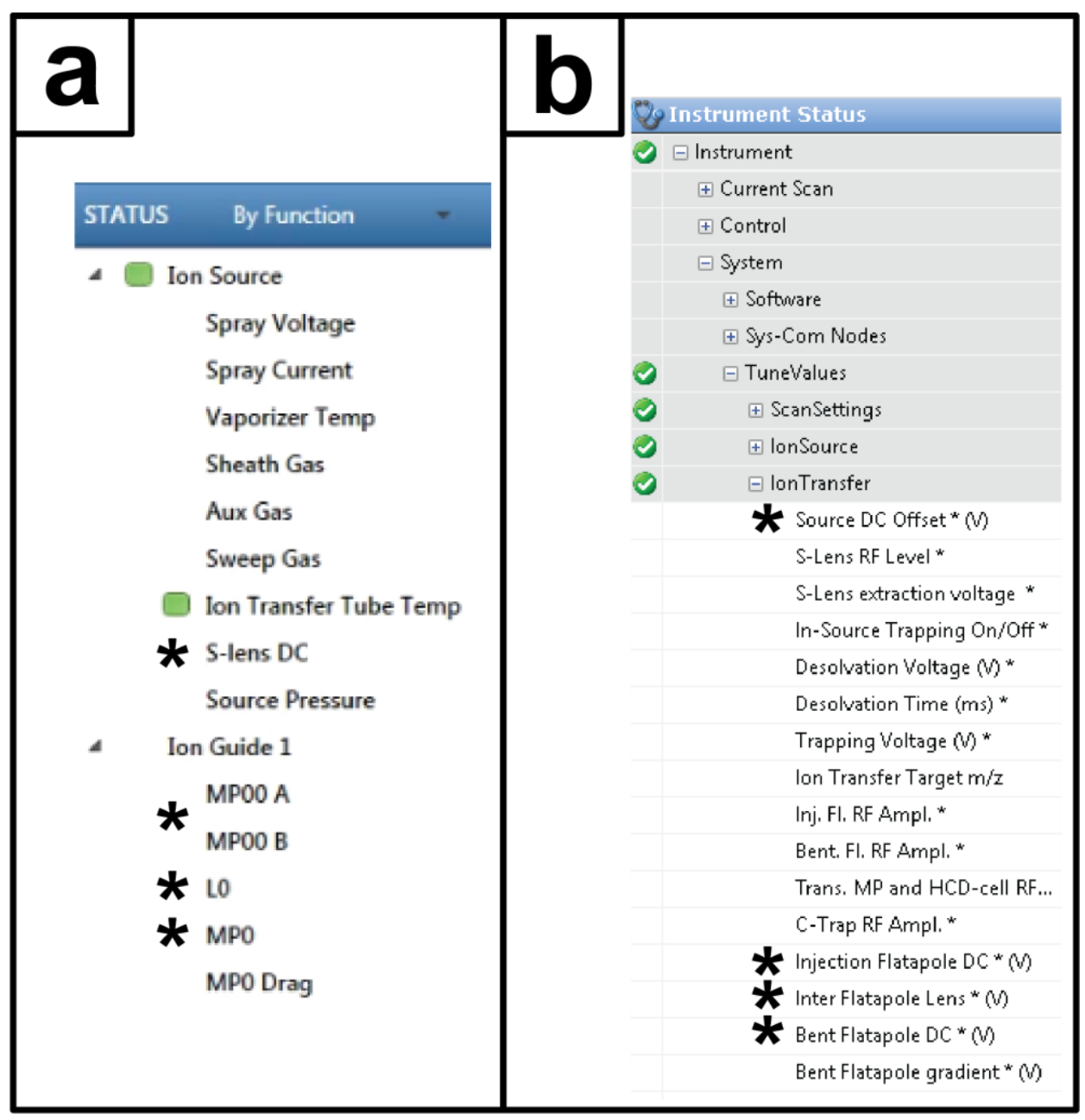

Fig. S2. Tune tree examples with marked pertinent voltages. Asterisks denote parameters of interest with respect to VRF. a) Tune tree of an Orbitrap Fusion Lumos. b) Tune tree of a Q-Exactive UHMR. Note that a developer's license is required to view and/or alter the pertinent parameters. 
Table S1. Nomenclature and default values used for testing.

\begin{tabular}{|c|c|c|}
\hline Tribrid Nomenclature & $\begin{array}{c}\text { "Default" Eclipse } \\
\text { (Tribrid) Values Used }\end{array}$ & $\begin{array}{c}\text { "Default" Lumos (Tribrid) } \\
\text { Values Used }\end{array}$ \\
\hline S-lens DC (SRIG) & $20.1 \mathrm{~V}$ & $23.7-37.6 \mathrm{~V}$ \\
\hline MP0O DC & $8.1 \mathrm{~V}$ & $11.0-11.5 \mathrm{~V}$ \\
\hline LO DC & $7.3-7.4 \mathrm{~V}$ & $7.6-7.8 \mathrm{~V}$ \\
\hline MPO DC & $6.3 \mathrm{~V}$ & $6 \mathrm{~V}$ \\
\hline MP0O RF Amplitude & $530 \mathrm{~V}$ & $430 \mathrm{~V}$ \\
\hline MPO RF Amplitude & $800 \mathrm{~V}$ & $640 \mathrm{~V}$ \\
\hline MP00/MP0 RF Frequency & $2.1 \mathrm{MHz}$ & $2.8 \mathrm{MHz}$ \\
\hline Source Pressure & $2.2-2.3 \mathrm{Torr}$ & $2.5-2.6 \mathrm{Torr}$ \\
\hline
\end{tabular}



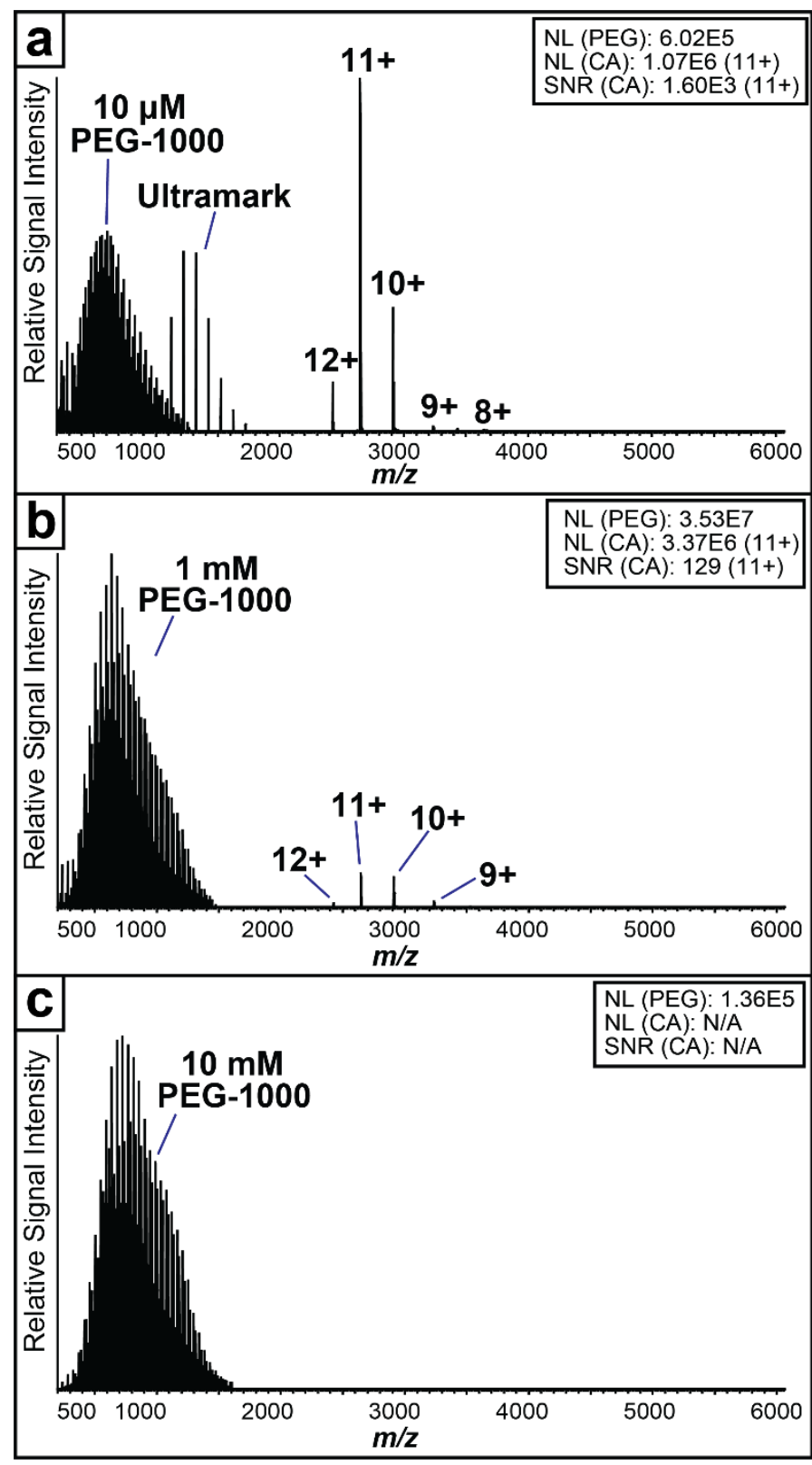

Fig. S3. Spectra of carbonic anhydrase at multiple levels of adulteration. Spectra of $11 \mu \mathrm{M}$ carbonic anhydrase in $100 \mathrm{mM}$ ammonium acetate were acquired using $10 \mu$ scans, $\sim 20$-scan averages, and injection times of $5 \mathrm{~ms}$. Data were taken on an Orbitrap Fusion Lumos. Default voltage values were used. a) $10 \mu \mathrm{M}$ PEG-1000. b) 1 mM PEG-1000. c) 10 mM PEG-1000. 
Table S2. Average Extracted Ion Current (EIC) and Total Ion Current (TIC) of presented figures.

\begin{tabular}{|c|c|c|c|}
\hline Figure & & Average EIC & Average TIC \\
\hline \multirow{4}{*}{ Figure 2} & a & $\mathrm{N} / \mathrm{A}$ & $1.00 \mathrm{E}+09$ \\
\hline & b & $2.6 \mathrm{E} 3(23+)$ & $1.40 \mathrm{E}+06$ \\
\hline & c & N/A & $2.20 \mathrm{E}+05$ \\
\hline & d & $1.1 \mathrm{E} 3(23+)$ & $1.40 E+04$ \\
\hline \multirow{2}{*}{ Figure 3} & b & $4.8 \mathrm{E} 4(23+$, dominant proteoform $)$ & $3.70 \mathrm{E}+05$ \\
\hline & d & 3.0E3 (33+, dominant proteoform) & $2.20 \mathrm{E}+04$ \\
\hline \multirow{2}{*}{ Figure $\mathbf{S 2}$} & a & N/A & $1.30 \mathrm{E}+06$ \\
\hline & b & 1.1E3 $(24+$, monomer $)$ & $1.40 E+04$ \\
\hline \multirow{3}{*}{ Figure S6 } & a & $1.6 \mathrm{E} 3(11+)$ & $7.50 \mathrm{E}+07$ \\
\hline & b & 3.4E6 (11+) & $3.60 E+09$ \\
\hline & c & N/A & $1.60 \mathrm{E}+07$ \\
\hline
\end{tabular}




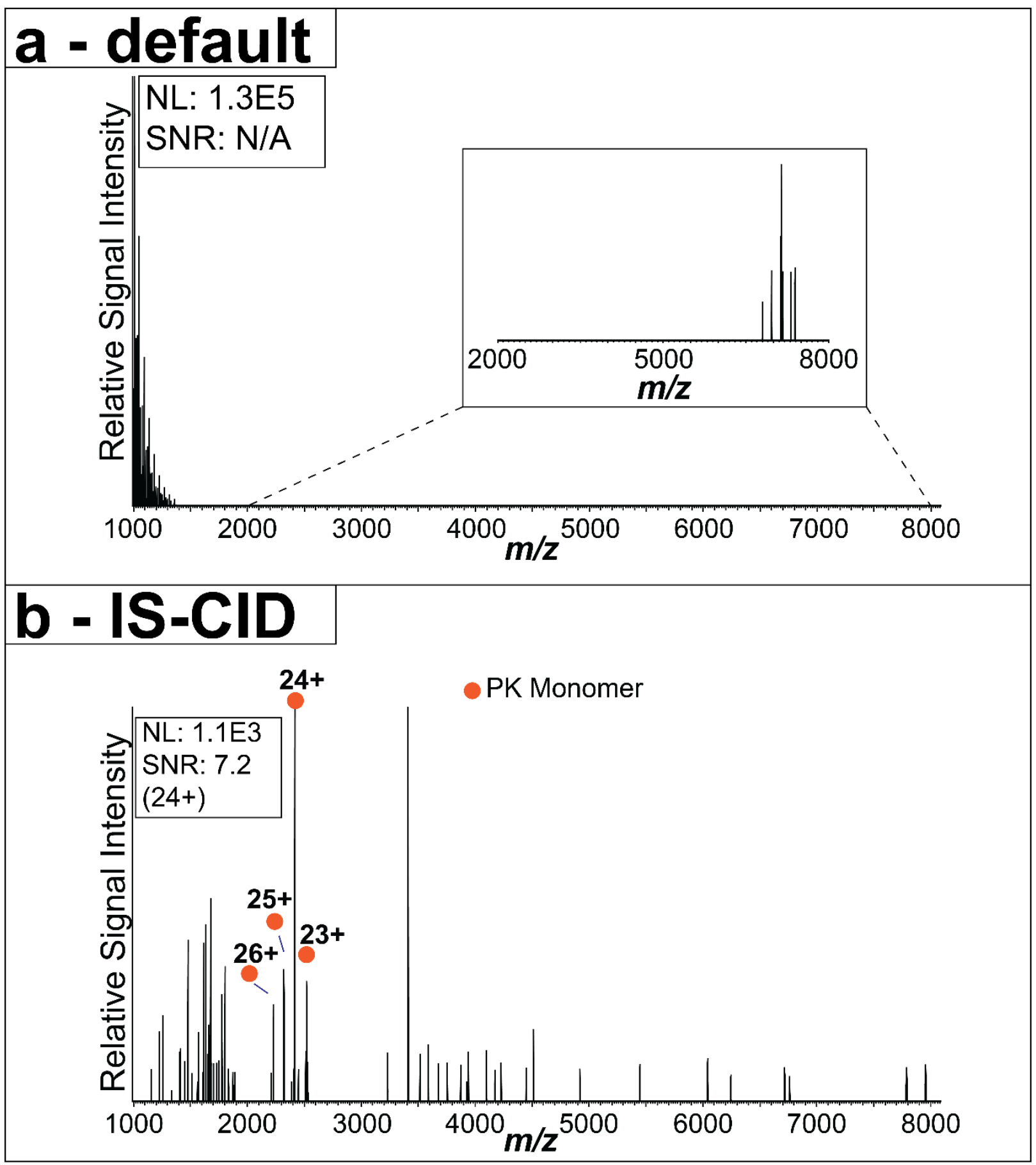

Fig. S4. Observed PEG space-charge effects on pyruvate kinase. Pyruvate kinase was sprayed at $10 \mu \mathrm{M}$ and adulterated with $2 \mathrm{mM}$ PEG-1000. a) Under standard settings, there is no discernable pyruvate kinase signal. b) Incorporation of 200 V IS-CID drastically attenuates the polymer adulterant but completely ejects the monomer from the analyte complex. See Table S2 for additional ion currents. 


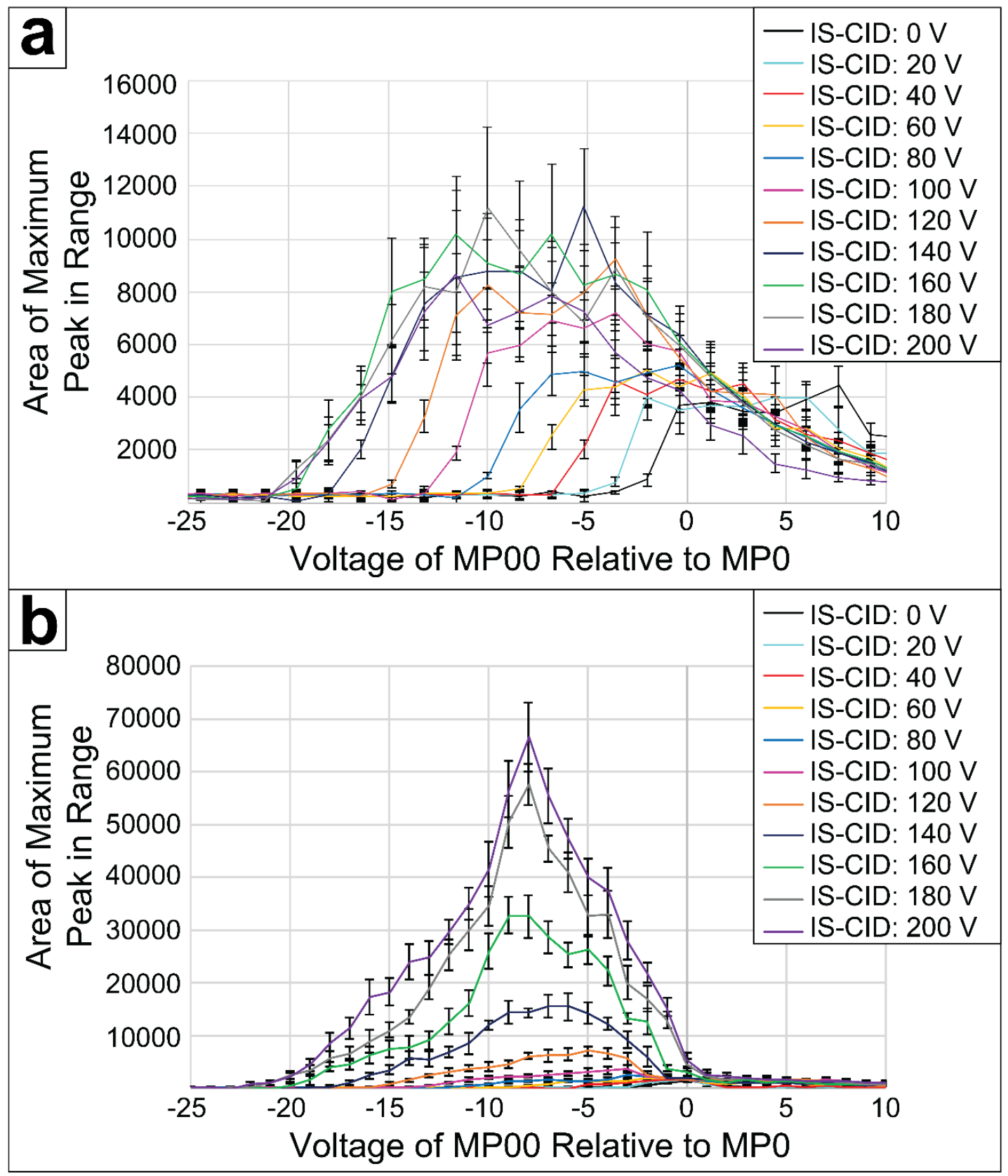

Fig. S5. Ramped optimizations of adulterated and unadulterated SILu ${ }^{\mathrm{TM}}$ MAb Antibody. Each data point is the average of 10 replicates, and the error bars indicate standard deviations. a) Signal maxima for unadulterated antibody shift depending on the in-source collision-induced dissociation (IS-CID).b) Signal maxima for antibody adulterated with $2 \mathrm{mM}$ PEG-1000 have sharper profiles and have greater dependence on source activation voltage. Note that peak area is used as a self-contained measure and is not rigorously structured as a quantitative measure for unadulterated vs. filtered samples due to differences in spray conditions and spectral quality. 


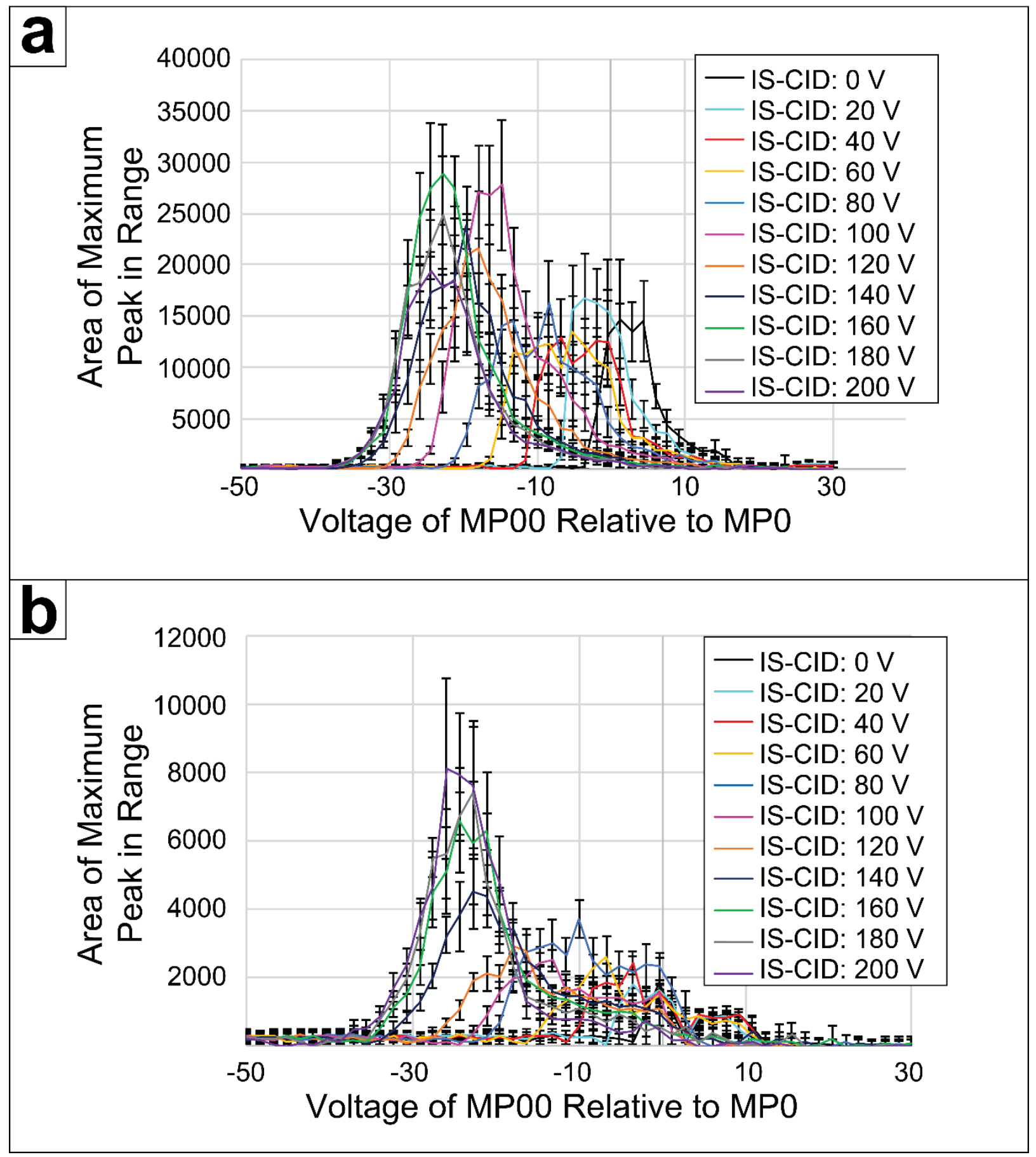

Fig. S6. Ramped optimizations of adulterated and unadulterated, tetrameric pyruvate kinase. Each data point is the average of 10 replicates. a) Unadulterated sample. b) Adulterated sample of pyruvate kinase containing $2 \mathrm{mM}$ PEG-1000. Note that peak area is used as a self-contained measure and is not rigorously structured as a quantitative measure for unadulterated vs. filtered samples due to differences in spray conditions and spectra quality. 


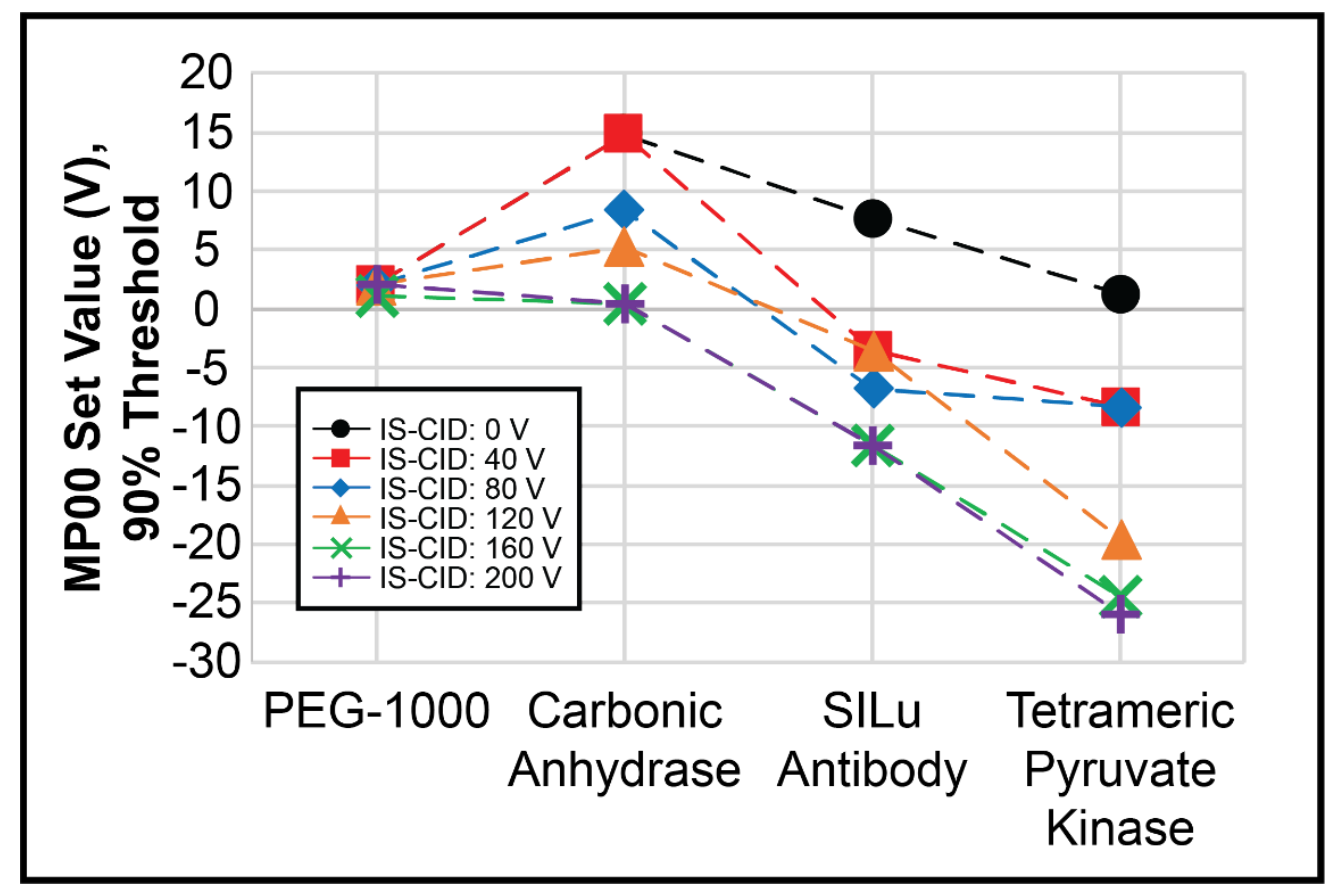

Figure S7. Favorable MP00 values by native species and IS-CID activation. Data points denote the lowest MP00 value where the corresponding analyte reached $90 \%$ of its maximum during optimization. Dashed lines are included for clarity in the cases where data points overlap. Note that voltage ramping was conducted in discrete steps. 


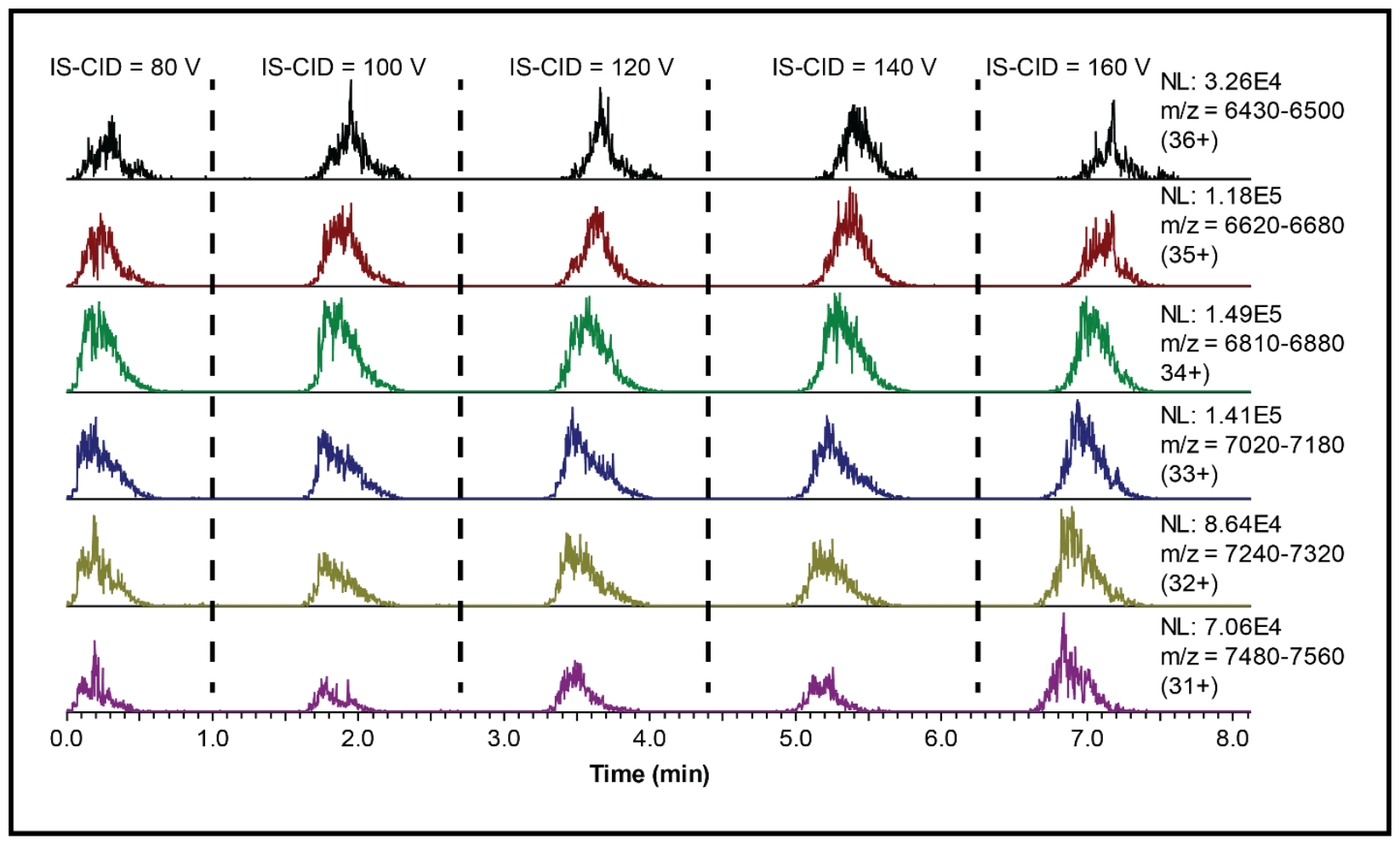

Figure S8. Extracted Ion Current for unadulterated, tetrameric pyruvate kinase during voltage optimization ramps. For a set ISCID (marked off in regions of time by the dashed lines), the MP00 and L0 values were ramped from their minimum set values to their maximum set values (see Experimental section). 


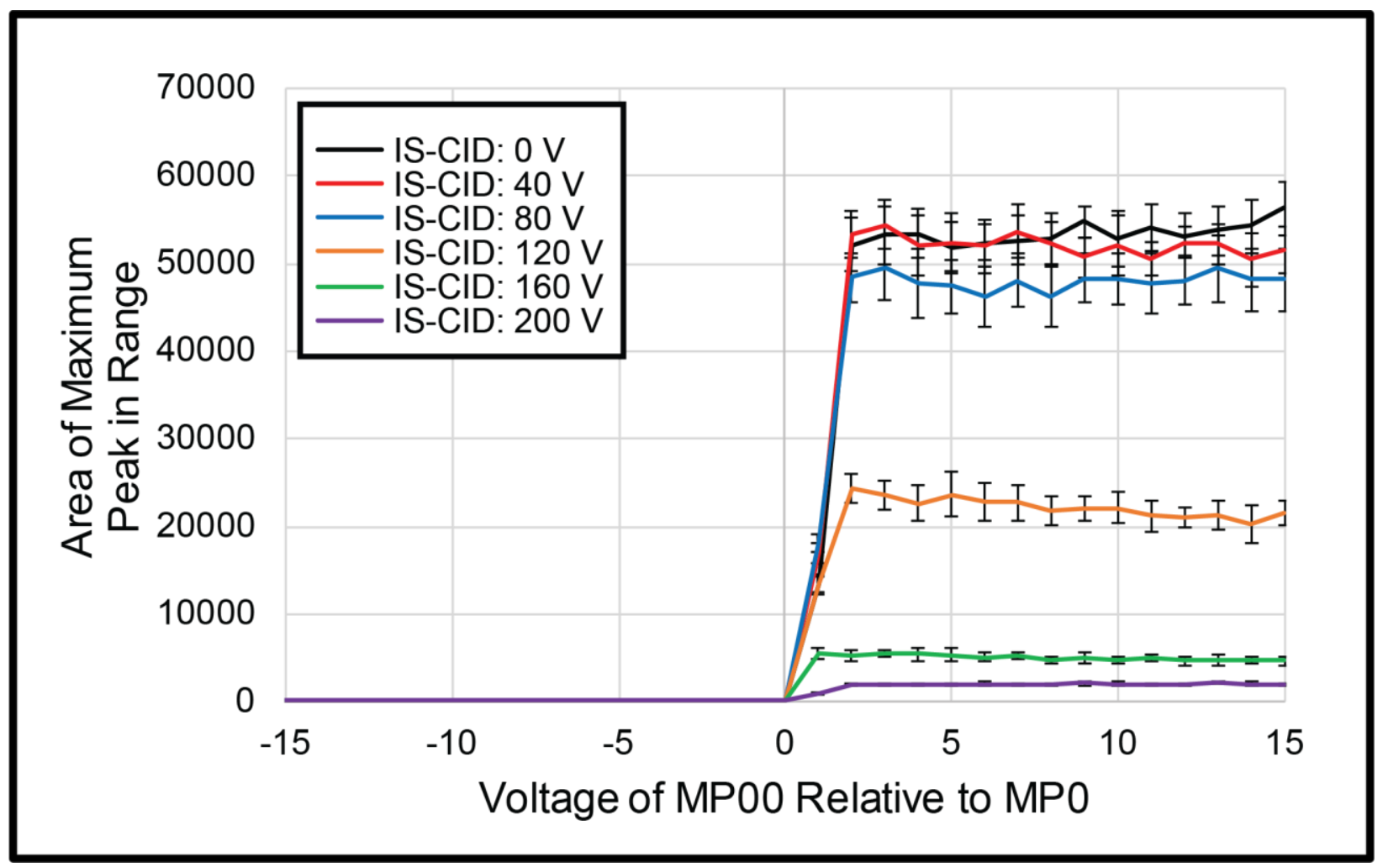

Fig. S9. Ramped optimizations of the adulterant PEG-1000. Each data point was the average of 5 replicates, and the error bars indicated standard deviations; data were from running a solution of $5 \mu \mathrm{M}$ PEG-1000 on an Orbitrap Fusion Lumos. 


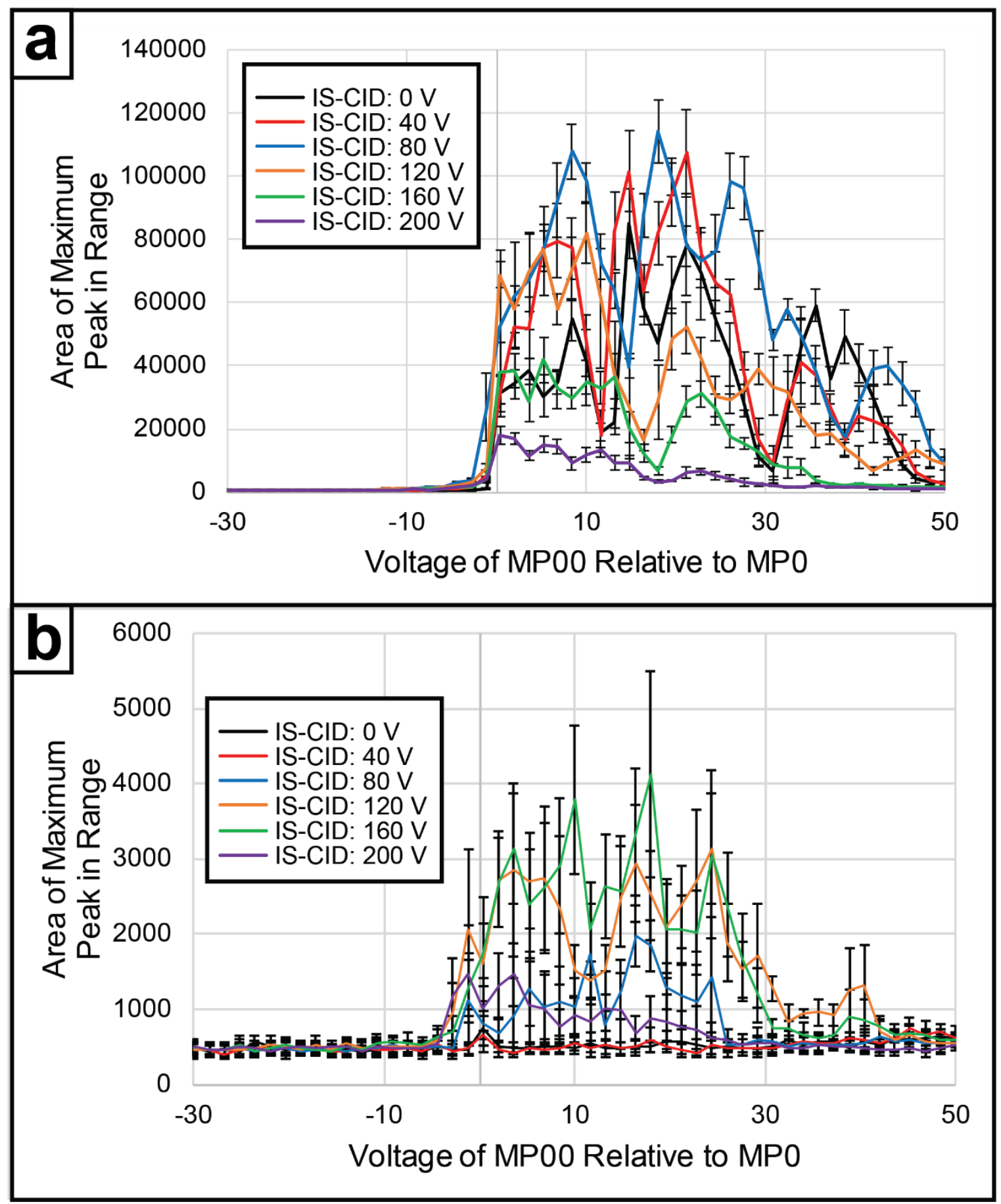

Fig. S10. Ramped optimizations of adulterated and unadulterated, carbonic anhydrase. Data were taken on an Orbitrap Fusion Lumos $(\mathrm{n}=0)$. a) Unadulterated sample. b) Adulterated sample of carbonic anhydrase containing 10 mM PEG-1000. Note that peak area is used as a self-contained measure and is not rigorously structured as a quantitative measure for unadulterated vs. filtered samples due to differences in spray conditions and spectra quality. 


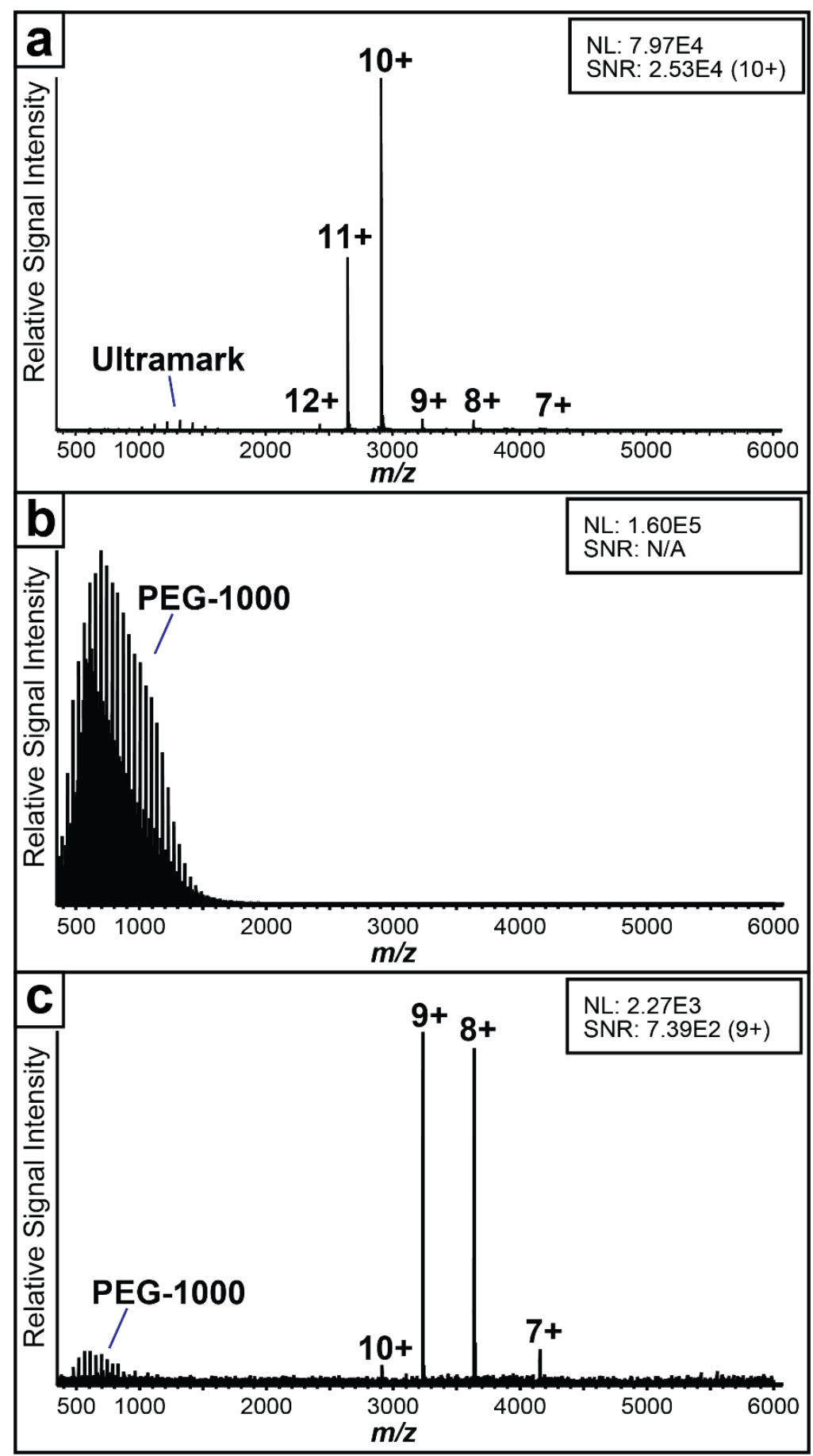

Fig. S1 1. Spectra of unadulterated and adulterated but filtered carbonic anhydrase. Spectra of $10 \mu \mathrm{M}$ carbonic anhydrase in $100 \mathrm{mM}$ ammonium acetate were acquired using $1 \mu$ scan, 20-scan averages, and injection times of $5 \mathrm{~ms}$. Data were taken on an Orbitrap Fusion Lumos. a) optimized and unadulterated ( $80 \mathrm{~V}$ for IS-CID, $8.5 \mathrm{~V}$ at MP00, and $6.5 \mathrm{~V}$ at L0). b) adulterated and not rollercoaster filtered (10 mM PEG-1000). Default values. c) adulterated sample with rollercoaster filtering (10 mM PEG-1000). $180 \mathrm{~V}$ for IS-CID, -1 V at MP00, and $-3 \mathrm{~V}$ at L0. See Table S2 for additional ion currents. 